\title{
Cristina Nanni, Stefano Fanti and Lucia Zanoni (eds): Radiology for PET/CT Reporting
}

\author{
Springer, Berlin 2014, ISBN: 978-3-642-40293-7
}

\author{
Sara M. d. A. Giorgio • Luigi Mansi
}

Published online: 31 July 2014

(C) Springer-Verlag Berlin Heidelberg 2014

The use of PET/CT is increasingly becoming routine in many institutions, particularly in the field of oncology; nevertheless, it still represents a challenge to correctly evaluate either the functional or the morphostructural component. In particular, many noteworthy normal, paraphysiological and benign pathological findings may be seen on CT images, although functionally silent on PET images. To gain valuable clinical information, these data must be reported, interpreted and compared to previous scans. Moreover, the CT scans obtained by hybrid imaging are acquired in a low-dose modality and they are thicker than diagnostic $\mathrm{CT}$.

The authors are recognized experts working in the Department of Nuclear Medicine of the University Hospital Sant'Orsola Malpighi of Bologna, an institution that played and continues to play a prominent role in the development of PET imaging, either with FDG and beyond FDG.

This "original" book of 150 pages, featuring more than 500 figures, focuses on CT images from standard PET/CT acquisitions. More correctly, this publication can be considered a casebook more than an atlas. In fact only the first chapter, concerning "normal anatomy", is organized as an atlas, identifying all the relevant anatomical structures that are of clinical importance in the standard diagnostic slices. Conversely, the other chapters, analysing head and neck, thorax, abdomen, pelvis and musculoskeletal system, are organized according to the collection of cases with common and uncommon FDGnegative anatomical abnormalities; the didactic value is assured by the addition of the main clinical applications, which are clearly articulate learning points and pitfalls.

Unfortunately, this book is, in a few cases, affected by a minor weakness, due to images that are sometimes too small, even if clearly printed and accompanied by teaching points easily understandable. A further improvement is given by the association with information on imaging techniques and the meaning of the explained parameters provided. An easy solution for an improvement could be to add a website connection, giving the possibility to magnify images and/or to further increase the number of them to better understand some individual cases.

Our opinion on this book remains certainly positive, also because it serves to occupy an almost empty space with a handy, clear and simple publication. We suggest this atlas as a standard text for nuclear medicine physicians and radiologists, residents and technicians whose work involves PET/CT imaging. It can also be suitable as a text for both undergraduate and postgraduate courses.

S. M. d. A. Giorgio · L. Mansi ( $\square)$

Second University of Naples, Naples, Italy

e-mail: luigi.mansi@unina2.it 